21st Particles and Nuclei International Conference (PANIC 2017)

International Journal of Modern Physics: Conference Series

Vol. 46 (2018) 1860046 (6 pages)

(C) The Author(s)

DOI: $10.1142 / \mathrm{S} 2010194518600467$

\title{
Dark Photon Searches at BESIII
}

\author{
Dayong Wang (for the BESIII Collaboration) \\ School of Physics and State Key Lab. of Nucl. Phys. and Tech., \\ Peking University, Beijing 100871, China \\ dayong.wang@pku.edu.cn
}

Published 3 May 2018

\begin{abstract}
Many models beyond the Standard Model, motivated by the recent astrophysical anomalies, predict a new type of weak-interacting degrees of freedom. Typical models include the possibility of the low-mass dark gauge bosons of a few $\mathrm{GeV}$ and thus making them accessible at the BESIII experiment running at the tau-charm region. The BESIII has recently searched such dark bosons in several decay modes using the high statistics data set collected at charmonium resonaces. This talk will summarize the recent BESIII results of these dark photon searches and related new physics studies.
\end{abstract}

Keywords: BESIII; dark photon; new physics.

\section{Introduction}

BEPCII is the only currently running $\tau$-charm factory working at the c. m. energy range of 2.0-4.6GeV, located at Institute of High Energy Physics, Beijing. This energy range has a lot of unique features which benefit greatly the rich physics programs.

The BESIII detector has a geometrical acceptance of $93 \%$ of $4 \pi$ and consists a small-celled, helium-based main draft chamber, an electromagnetic calorimeter, a time-of-flight system for particle identification, a muon chamber system(MUC) made of about $1272 \mathrm{~m}^{2}$ of resistive plate chambers incorpared in the return iron of the superconducting solenoid with $1 \mathrm{~T}$ magentic field. More details of the detector are described in Ref ${ }^{1}$.

BESIII has accumulated 1.3 Billion $J / \psi$ 's, 0.5 Billion $\psi(3686)$ 's and $2.9 \mathrm{fb}^{-1}$ at $\psi(3773)$, all of which are the largest data sets in the world. In general, the clean environments and high luminosity at BESIII provide ideal samples for searches for new physics beyond standard model.

This is an Open Access article published by World Scientific Publishing Company. It is distributed under the terms of the Creative Commons Attribution 4.0 (CC-BY) License. Further distribution of this work is permitted, provided the original work is properly cited. 


\section{Wang}

Many models beyond the Standard Model predict a new type of weak-interacting degree of freedom ${ }^{2}$. The dark photon (DP, denoted as $\gamma^{\prime}$ or $\mathrm{U}$ ) is a new Abelian gauge group $\mathrm{U}(1)$ force carrier, which couples to the SM via kinetic mixing ${ }^{3}$ with a mixing strength. It has been proposed to explain the large number of astrophysical anomalies ${ }^{4}$, as well as the deviation observed in the anomalous magnetic momentum of muon ${ }^{5}$.

Typical models include the possibility of the low-mass DPs of a few GeV and thus making them accessible at the BESIII experiment running at the tau-charm region. The BESIII has recently searched such dark bosons using the high statistics data set collected at charmonium resonances. DP at BESIII is searched with two different categories of methods: with untagged ISR events and from charmonium decays.

\section{DP search through untagged ISR events ${ }^{6}$}

The search with ISR events is perfoprmed with $2.93 \mathrm{fb}^{-1}$ data sample taken at the center of mass energy $\sqrt{s}$ of $3.773 \mathrm{GeV}^{7}$. ISR reactions $e^{+} e \rightarrow \gamma_{I S R} e^{+} e^{-}$and $e^{+} e \rightarrow \gamma_{I S R} \mu^{+} \mu^{-}$are used, in which one of the beam particles radiates a photon. In this way, the mass region from the final state particles mass threshold to $\mathrm{s}$ is accessible. The weak coupling of the DP implies that the decay width of the DP would be far smaller than the resolution of the experiment, resulting a sharp peak on top of the ISR QED background events. The invariant mass spectrum of the lepton pair from data is fitted with a 4th order polynomial function, and the difference between data spectrum and the fitted curve is used to test the existence of the DP.

The $\mu^{+} \mu^{-}$and $e^{+} e^{-}$invariant mass distributions, $m_{\mu^{+} \mu^{-}}$and $m_{e^{+} e^{-}}$, which are shown separately in Fig. 1, are mainly dominated by the QED background but could contain the signal sitting on top of these irreducible events. For comparison with data, MC simulation, scaled to the luminosity of data, is shown, although it is
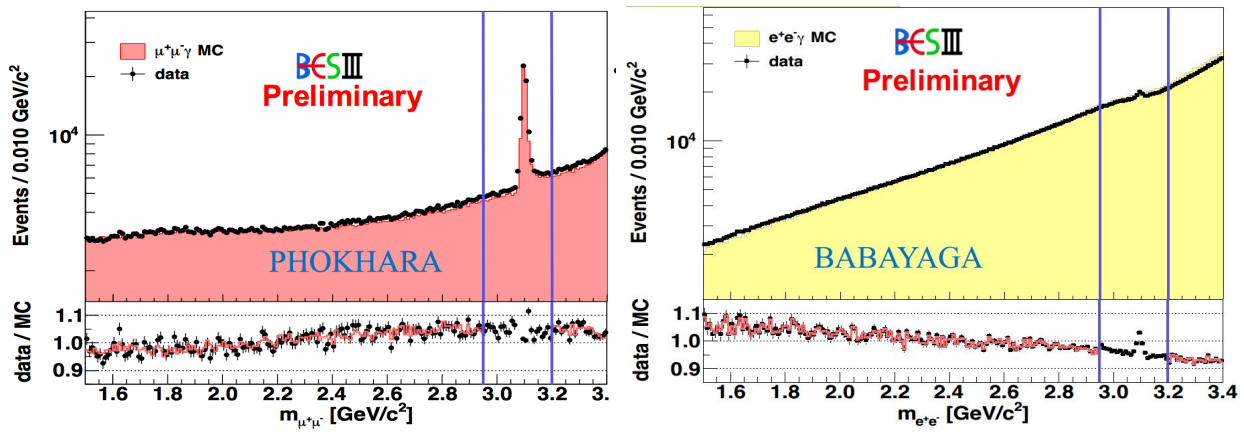

Fig. 1. Leptonic invariant mass distributions $m_{\mu^{+} \mu^{-}}$and $m_{e^{+} e^{-}}$after applying the selection requirements. Shown is data (points) and MC simulation (shaded area), which is scaled to the luminosity of the data set. The marked area around the $\mathrm{J} /$ resonance is excluded in the analysis. The lower panel shows the ratio of data and MC simulation (points) and the ratio of fit curve and MC simulation (histogram). 

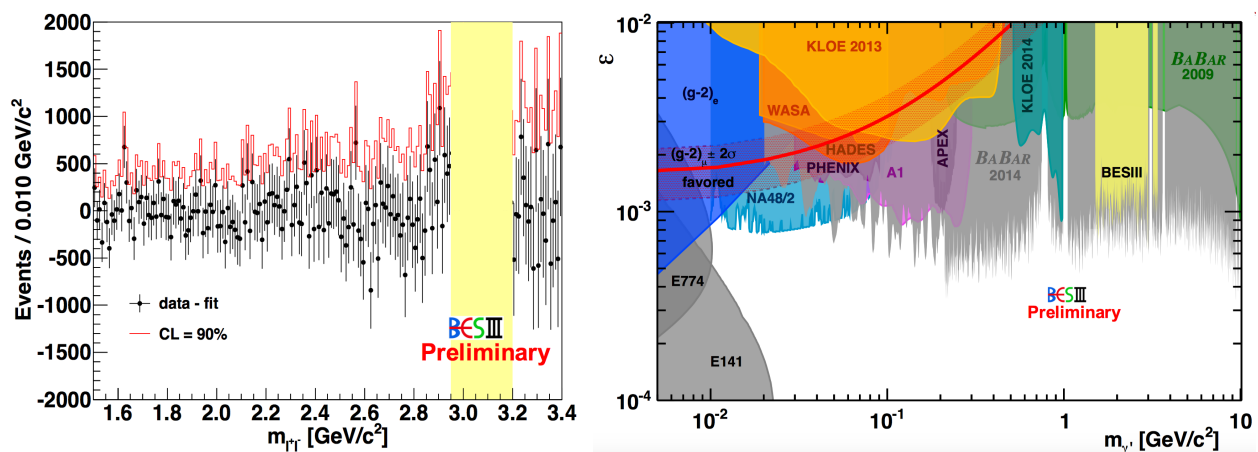

Fig. 2. Results from DP search from ISR events. Left figure: The sum of the differences between the ISR event yields and their respective 4th order polynomials (dots with error bars). The red solid histogram represents the exclusion limit with the $90 \%$ confidence, cal- culated with a profile likelihood approach and including the systematic uncertainty. The region around the $J / \psi$ resonance between 2.95 and $3.2 \mathrm{GeV} / \mathrm{c}^{2}$ is excluded. Right figure: Exclusion limit at the $90 \%$ confidence level on the mixing parameter $\epsilon$ as a function of the DP mass. The bold solid line represents the $\epsilon$ values, which would explain the discrepancy between the measurement and the SM calculation of the anomalous magnetic moment of the muon, together with its $2 \sigma$ band.

not used in the search for the DP. In this analysis, the dark photon mass range $m_{\gamma^{\prime}}$ between 1.5 and $3.4 \mathrm{GeV} / \mathrm{c}^{2}$ is studied. Below $1.5 \mathrm{GeV} / \mathrm{c}^{2}$ the $e^{+} e \rightarrow \gamma_{I S R} \pi^{+} \pi^{-}$ cross section with muon misidentification dominates the $m_{\mu^{+} \mu^{-}}$spectrum. Above $3.4 \mathrm{GeV} / \mathrm{c}^{2}$ the hadronic $q \bar{q}$ process can not be suppressed sufficiently by the $\chi_{1 C}^{2}$ requirement. In order to search for narrow structures on top of the QED background, 4th order polynomial functions to describe the continuum QED are fitted to the data distributions shown in Fig. 1. The mass range around the narrow $J / \psi$ resonance between 2.95 and $3.2 \mathrm{GeV} / \mathrm{c}^{2}$ is excluded.

The results from two final states are combined. No peaking structure is observed, and the statistical significance is less than 3 everywhere in the explored region. Thus the upper limit on the number of events at $90 \%$ confidence level (C.L.) is calculated using profile likelihood approach with systematic uncertainty included. shown in Fig. 2(left) shows the bin-by-bin calculated exclusion limit as a function of $m_{l^{+} l^{-}}$, including the systematic uncertainties.

The kinetic mixing parameter $\epsilon$ is calculated bin-by-bin with Eq.(19) from Ref. ${ }^{8}$. The upper limits on the mixing strength at $90 \%$ C.L. is determined between $10^{-3}$ and $10^{-4}$ depending on the dark photon mass, as shown in Fig. 2 (right). The BESIII result based on 2 years of data taking is competitive comparing to the best upper limit in this region from BaBar $^{9}$ based on 9 years running.

\section{DP searches from charmonium decays}

The electromagnetic (EM) Dalitz decay $V \rightarrow \mathrm{Pl}^{+} l^{-}$, where $V$ refers to a vector meson and $P$ a pseudoscalar meson, provides important information on the 
interactions at the $V-P$ transition vertex ${ }^{10}$. This process was first observed by BESIII in $2014^{11}$ and is predicted to be sensitive for DP search ${ }^{12}$.

BESIII has performed a DP search through $J / \psi \rightarrow \eta^{\prime} \mathrm{U}$ with a data set of $(1310.6 \pm 7.0) \times 10^{6} \mathrm{~J} / \psi$ events $^{13}$. The Feynman diagram is shown in Fig. 3 . It is the first time that $\mathrm{DP}$ is searched through charmonium decay $J / \psi \rightarrow \eta^{\prime} \mathrm{U}, \mathrm{U} \rightarrow e^{+} e^{-}$.

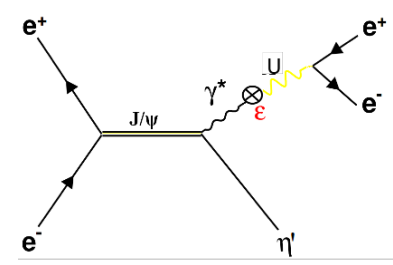

Fig. 3. Feynman diagram for the process of $J / \psi \rightarrow P U, U \rightarrow e^{+} e^{-}$.

The $\eta^{\prime}$ meson is reconstructed with the two decay modes $\eta^{\prime} \rightarrow \gamma \pi^{+} \pi^{-}$(modeI) and $\eta \rightarrow \pi^{+} \pi^{-} \eta(\gamma \gamma)$ (mode-II) in the dark photon search. The reconstructed particles in the final state are $\gamma \pi^{+} \pi^{-} e^{+} e^{-}$and $\gamma \gamma \pi^{+} \pi^{-} e^{+} e^{-}$, respectively.

Due to dominance of $\gamma$ conversion events, we discard the events with $M\left(e^{+} e^{-}\right)<$ $70 \mathrm{MeV} / c^{2}$. Electron-positron from decays of vector mesons $\omega, \phi$ will contaminate the possible DP signal and significantly suppresses the lepton decay mode of DP ${ }^{15}$. Therefore, the mass range $[0.74,0.84] \mathrm{GeV} / c^{2}$ and $[1.00,1.04] \mathrm{GeV} / c^{2}$, corresponding to the region of vector meson $\omega$ and $\phi$, are excluded in the DP search.

A series of signal MC are generated according to decay chain $J / \psi \rightarrow \eta^{\prime} \mathrm{U}, \mathrm{U} \rightarrow$ $e^{+} e^{-}$, with different DP mass $m_{\mathrm{U}}$ hypotheses, ranging from 0.1 to $2.0 \mathrm{GeV} / c^{2}$ with step of $0.1 \mathrm{GeV} / c^{2}$. The DP signal PDF is parametrized by the sum of two Crystal Ball (CB) functions with common mean value. The resolution of $m_{\mathrm{U}}$ grows from 1 $\mathrm{MeV} / c^{2}$ to $8 \mathrm{MeV} / c^{2}$ as the $m_{\mathrm{U}}$ gradually becomes larger. The detection efficiency ranges from $35 \%$ to $41 \%$ ( $22 \%$ to $27 \%$ ) depending on the $m_{\mathrm{U}}$ in mode I (II).

A series of ML fit are performed on the $M\left(e^{+} e^{-}\right)$spectra with $M_{\mathrm{U}}$ at the assumption from 0.1 to $2.0 \mathrm{GeV} / c^{2}$ on step of $2 \mathrm{MeV} / c^{2}$ to extract the yield of possible DP signals. The signal efficiency and PDF parameters are interpolated between mass points from a polynomial fitting. The shapes of the signal and background PDFs are fixed while the numbers of signal and background events are left free in the fit.

The maximum local significance is $3.1 \sigma$ at $0.204 \mathrm{GeV} / c^{2}$, with corresponding global significance less than $1 \sigma$. Thus, no significant DP signal is observed within the searched range. So we compute the upper limit on the branching fraction $\mathcal{B}(J / \psi \rightarrow$ $\left.\eta^{\prime} \mathrm{U}\right)$ at $90 \%$ C.L. using a Bayesian method. The observed number of signal events in $i^{\text {th }}$ mode is calculated with $N_{\text {sig }}^{i}=N_{J / \psi} \cdot \mathcal{B}_{\eta^{\prime} \rightarrow \mathrm{F}}^{i} \cdot \mathcal{B}_{J / \psi \rightarrow \mathrm{U} \eta^{\prime}} \cdot \mathcal{B}_{\mathrm{U} \rightarrow e^{+} e^{-}} \cdot \mathscr{E} i$, where $N_{J / \psi}$ is the total number of $J / \psi$ events, $\mathcal{B}_{\eta^{\prime} \rightarrow \mathrm{F}}^{i}$ is the branching fraction of $\eta^{\prime}$ decay to final states $\mathrm{F}, \mathcal{B}_{J / \psi \rightarrow \mathrm{U} \eta^{\prime}}$ and $\mathcal{B}_{\mathrm{U} \rightarrow e^{+} e^{-}}$are the branching fractions that corresponding subscript denotes and $\mathscr{E}^{i}$ is the detection efficiency determined from signal MC. The likelihood $(\mathcal{L})$, as a function of product branching fraction $\mathcal{B}(J / \psi \rightarrow$ 
$\left.\eta^{\prime} \mathrm{U}\right) \times \mathcal{B}\left(\mathrm{U} \rightarrow e^{+} e^{-}\right)$, is calculated as a product of $\mathcal{L}$ from mode-I and modeII with method described in Ref. ${ }^{14}$. The systematic uncertainties are separately incorporated into the likelihood as correlated and uncorrelated sources. The upper limit $\mathcal{B}^{\mathrm{UP}}$ on the product branching fraction at the $90 \%$ C.L. are determined from integral $\int_{0}^{\mathcal{B}^{\mathrm{UP}}} \mathcal{L} d \mathcal{B} / \int_{0}^{\infty} \mathcal{L} d \mathcal{B}=90 \%$.

The values of $\mathcal{B}^{\mathrm{UP}}$ is plotted as a function of $m_{\mathrm{U}}$ as shown in Fig. 4 (left top). The product branching fraction of $\mathcal{B}\left(J / \psi \rightarrow \eta^{\prime} \mathrm{U}\right) \times \mathcal{B}\left(\mathrm{U} \rightarrow e^{+} e^{-}\right)$is then divide by the theoretical branching fraction of $\mathcal{B}_{\mathrm{U} \rightarrow e^{+} e^{-}}$from Ref. ${ }^{15}$ to compute the upper limit on the branching fractions $\mathcal{B}\left(J / \psi \rightarrow \eta^{\prime} \mathrm{U}\right)$ at $90 \%$ C.L. and the corresponding result is shown in Fig. 4 (left bottom).
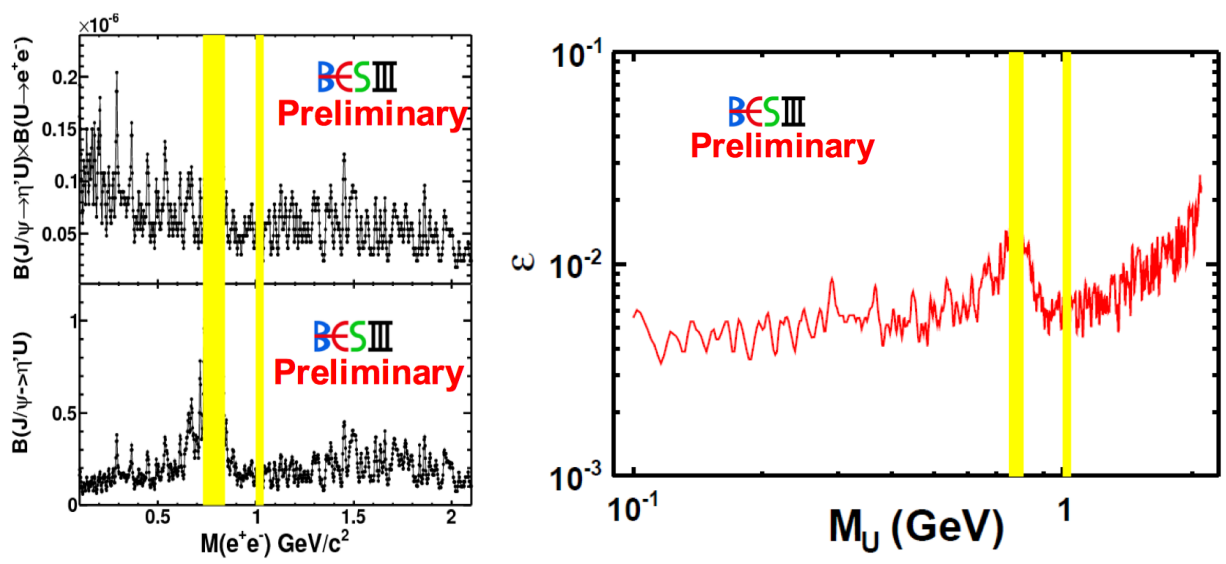

Fig. 4. Results from etap ee search. Left: Upper limit at $90 \%$ C.L. for the branching fraction of (top) $\mathcal{B}\left(J / \psi \rightarrow \eta^{\prime} \mathrm{U}\right) \times \mathcal{B}\left(\mathrm{U} \rightarrow e^{+} e^{-}\right)$and (bottom) $\mathcal{B}\left(J / \psi \rightarrow \eta^{\prime} \mathrm{U}\right)$. Right: Experimental upper limit on the mixing strength $\varepsilon$ as a function of DP mass $m_{\mathrm{U}}$ at $90 \%$ C.L. from this work

The mixing strength $\varepsilon$ is extracted by the normalization between branching fraction of $\mathcal{B}\left(J / \psi \rightarrow \eta^{\prime} \mathrm{U}\right)$ and corresponding radiative process $\mathcal{B}\left(J / \psi \rightarrow \eta^{\prime} \gamma\right)$ as

$$
\frac{\mathcal{B}\left(J / \psi \rightarrow \eta^{\prime} \mathrm{U}\right)}{\mathcal{B}\left(J / \psi \rightarrow \eta^{\prime} \gamma\right)}=\varepsilon^{2}\left|F\left(m_{\mathrm{U}}^{2}\right)\right|^{2} \frac{\lambda^{3 / 2}\left(m_{J / \psi}^{2}, m_{\eta^{\prime}}^{2}, m_{\mathrm{U}}^{2}\right)}{\lambda^{3 / 2}\left(m_{J / \psi}^{2}, m_{\eta^{\prime}}^{2}, 0\right)},
$$

where $\lambda\left(m_{1}^{2}, m_{2}^{2}, m_{3}^{2}\right)=\left(1+\frac{m_{3}^{2}}{m_{1}^{2}-m_{2}^{2}}\right)^{2}-\frac{4 m_{1}^{2} m_{3}^{2}}{\left(m_{1}^{2}-m_{2}^{2}\right)^{2}}, \varepsilon$ is the kinematic mixing parameter, $m$ is mass of a specific particle, $\left|F\left(m_{\mathrm{U}}^{2}\right)\right|^{2}$ is the monopole form factor $J / \psi \rightarrow \eta^{\prime} e^{+} e^{-}$evaluated at $m_{\mathrm{U}}$. The branching fraction $\mathcal{B}\left(J / \psi \rightarrow \eta^{\prime} \gamma\right)$ is set at the PDG value ${ }^{16}$. The corresponding exclusion limit on mixing strength $(\varepsilon)$ and DP mass $\left(m_{\mathrm{U}}\right)$ is shown in Fig. 4(Right).

\section{Summary}

In summary, the clean environments, high luminosity and excellent detector performance at BESIII provide ideal opportunities for searches for new physics beyond 
standard model. We have searched dark photon in both ISR process and charmonium decays and more results will be coming.

We perform a search for a dark photon in the mass range between 1.5 and 3.4 $\mathrm{GeV} / \mathrm{c} 2$, where we do not observe a significant signal. We set upper limits on the mixing parameter $\epsilon$ between $10^{-3}$ and $10^{-4}$ as a function of the dark photon mass with a confidence level of $90 \%$. This is a competitive limit in this dark photon mass range. This is possible due to the use of untagged ISR events for the dark photon search as well as the fact that the center-of-mass energy of the BEPCII collider is closer to the mass region tested. We also use a different analysis approach, which has no dependence on the radiator function.

With a data sample of $(1310.6 \pm 7.0) \times 10^{6} \mathrm{~J} / \psi$ events collected by the BESIII detector, we search for a dark photon in $J / \psi$ decay with $J / \psi \rightarrow \eta^{\prime} \mathrm{U}, \mathrm{U} \rightarrow e^{+} e^{-}$ using the same two $\eta^{\prime}$ decay modes. No significant dark photon signal is observed and we set an upper limit for branching fraction product $\mathcal{B}\left(J / \psi \rightarrow \eta^{\prime} \mathrm{U}\right) \times \mathcal{B}\left(\mathrm{U} \rightarrow e^{+} e^{-}\right)$ and branching fraction $\mathcal{B}\left(J / \psi \rightarrow \eta^{\prime} \mathrm{U}\right)$ for the first time. The corresponding mixing strength $\epsilon$ between SM photon and dark photon under different dark photon mass $m_{U}$ is constrained.

\section{Acknowledgments}

This work is supported, in part, by Joint Funds of the National Natural Science Foundation of China (Grants No. U1232105) and by Ministry of Science and Technology (Grants No. 2015CB856700).

\section{References}

1. M. Ablikim et al. (The BESIII Collaboration), Nucl. Instrum. Meth. A 614, 345 (2010).

2. R. Essig et al., arXiv:1311.0029 and references therein.

3. B. Holdom, Phys. Lett. B 166, 196 (1986).

4. N. Arkani-Hamed, D. P. Finkbeiner, T. R. R. Skatyer, and N. Weiner, Phys. Rev. D 79, 015014 (2009); R. Essig, P. Schuster, and N. Toro, Phys. Rev. D 80, 015003 (2009).

5. M. Pospelov, Phys. Rev. D 80, 095002 (2009).

6. M. Ablikim et al. (BESIII Collaboration), arxiv:1705.04265, Phys. Lett. B in print.

7. M. Ablikim et al. (BESIII Collaboration), Phys. Lett. B 753, 629 (2016).

8. J. D. Bjorken, R. Essig, P. Schuster, and N. Toro, Phys. Rev. D 80, 075018 (2009).

9. J. P. Lees et al. (BABAR Collaboration), Phys. Rev. Lett. 113, 201801 (2014).

10. L. G. Landsberg, Phys. Rept. 128, 301 (1985).

11. M. Ablikim et al. (BESIII Collaboration), Phys. Rev. D 89, 092008 (2014).

12. J. Fu, H. B. Li, X. Qin, and M. Z. Yang, Mod. Phys. Lett. A 27, 1250223 (2012).

13. M. Ablikim et al. (BESIII Collaboration), Chin. Phys. C 41, 013001 (2017).

14. K. Stenson, arXiv:physics/0605236.

15. B. Batell, M. Pospelov and A. Ritz, Phys. Rev. D 79, 115008 (2009).

16. C. Patrignani et al. (Particle Data Group), Chin. Phys. C 40, 100001 (2016). 